\title{
Fitting orbits
}

\section{Andrzej J. Maciejewski ${ }^{1}$, Krzysztof Goździewski ${ }^{2}$ and Szymon Kozłowski ${ }^{1}$}

\author{
${ }^{1}$ Institute of Astronomy, University of Zielona Góra, Podgórna 50, PL-65-246 Zielona Góra, \\ Poland \\ email: maciejka@astro.ia.uz.zgora.pl \\ ${ }^{2}$ Toruń Centre for Astronomy, N. Copernicus University, Gagarina 11, PL-87-100 Toruń, \\ Poland
}

Nowadays, more than one hundred extra-solar planets are known, and about a dozen of multi-planetary systems have been discovered. Most of them have been detected by the radial velocity (RV) method. The recovery of orbital parameters from RV data leads to several problems. Usually RV data cover irregularly a short time interval which is frequently shorter than the orbital period of the most distant planet. Moreover, observations contain a noise due to the instabilities of the star. The distribution of this noise is unknown. A precise determination of the dynamical state of a multi-planetary system is important for understanding its stability and evolution. In most cases observers determine the orbital parameters for multi-planetary systems just fitting a sum of Keplerian orbits. The parameters obtained in such a way are in most cases the only accessible data about an extra-solar system because the observes very rarely publish their observations. However, the parameters from a multi-Keplerian fit as it has already been observed by many authors, cannot be interpreted as the osculating elements for actual planetary orbits. Moreover, these parameters can be considered as Keplerian elements of: relative, barycentric or Jacobi orbits. One can find arguments that the interpretation of parameters from a multi-Keplerian fit as elements of Keplerian orbits in the Jacobi coordinates is the most proper one, see [Lee and Peale, 2002; Goździewski et al. 2003].

Our first aim was to determine how badly a multi-Keplerian fit determines osculating orbits. To this end, we performed several numerical simulations. For a chosen planetary system with two planets we generated a synthetic RV observations using the Newtonian three body problem. Then we fitted to these observations the Keplerian model and compared the obtained Keplerian elements with the true osculating elements of orbits. Then we changed the semi-major axis and the eccentricity of one planet and repeated all calculations. In this way we obtained maps of differences between the true and the fitted Keplerian elements (relative, barycentric and Jacobi) for a given system with two planets.

The conclusions from these experiments are following. Even for a quite big separation of planets (2 AU), multi-Keplerian fits are bad. The errors appear mainly in the positions of planets in their orbits and can achieve $60 \mathrm{deg}$ and more. The errors in eccentricities and semi-major axes achieve a few percent, but they can be bigger for bigger masses of planets, or when the observations cover only a part of the period of the external planet. Moreover, the errors are maximal for systems close to a mean motions resonance. All the above conclusions do not depend on how we interprete the parameters of a Keplerian fit: relative, barycentric, as well as Jacobi elements are equally bad if we look at the overall results. 


\section{References}

Lee, M. H. and Peale, S. J. 2002, ApJ, 567, 596

Goździewski, K., Konacki, M. and Maciejewski, A. J. 2003, ApJ, 594, 1019 